\title{
Passenger Behavior Simulation in Congested Urban Rail Transit System: A Capacity-Limited Optimal Strategy Model for Passenger Assignment
}

\author{
Kai Lu $(\mathbb{D}$ and Nan Cao \\ Traffic Control Technology Co., Ltd., Beijing 100070, China \\ Correspondence should be addressed to Kai Lu; lukai_bjtu@163.com
}

Received 27 July 2021; Accepted 23 November 2021; Published 6 January 2022

Academic Editor: Jing-Hu Pan

Copyright (c) $2022 \mathrm{Kai} \mathrm{Lu}$ and Nan Cao. This is an open access article distributed under the Creative Commons Attribution License, which permits unrestricted use, distribution, and reproduction in any medium, provided the original work is properly cited.

\begin{abstract}
Optimal strategy, one of the main transit assignment models, can better demonstrate the flexibility for passengers using routes in a transit network. According to the basic optimal strategy model, passengers can board trains based on their frequency without any capacity limitation. In the metropolitan cities such as Beijing, Shanghai, and Hong Kong, morning commuters face huge transit problems. Especially for the metro system, there is heavy rush in metro stations. Owing to the limited train capacity, some passengers cannot board the first coming train and need to wait for the next one. To better demonstrate the behavior of passengers pertaining to the limited train capacity, we consider capacity constraints for the basic optimal strategy model to represent the real situation. We have proposed a simulation-based algorithm to solve the model and apply it to the Beijing Subway to demonstrate the feasibility of the model. The application of the proposed approach has been demonstrated using the computational results for transit networks originating from practice.
\end{abstract}

\section{Introduction}

Providing passenger-oriented service is the most important and core target for every metro system. To support passenger-friendly service planning and modification, it is necessary to have a passenger assignment model, which can demonstrate the passenger behavior and the distribution of passengers. Based on the assignment results, it would be clear to determine if the service is passenger friendly and how to provide a better service. Because of this, the assignment models are wildly used in trip planning process and the passenger departure time decision [1-3]. At the same time, when the disturbance or disruption happened, the operation scheme and the timetable could be adjusted according to the assignment results, such as retiming and providing short loop trains. In this way, the disorder could be solved with a more passenger-friendly solution [4-6].

Service frequency, which has high relation with system service quality and efficiency, is the key component for metro systems. The frequency is determined with the passenger demand, the passenger distribution, and operation costs. In some exurban places or low-demand areas, the transit service is quite poor and the train frequency is at the low level. Passengers must be informed with the accurate timetable, and they can schedule their departure time to catch the specific train service at the station. To demonstrate this precise passenger behavior and the interaction between passengers and trains, a schedule-based transit assignment approach is needed.

The schedule-based assignment is modeled based on a time-space network according to a timetable [7-10]. Similar to the frequency-based assignment, congestion is also an issue in the schedule-based network. Poon et al. [11] used a time-increment simulation to obtain the arrival and departure time of passengers to predict dynamic queuing delays and update the shortest path for the next simulation run. Nuzzolo et al. [12] defined a dynamic loading process on each transit run according to the user's choice and the 
residual capacity of vehicles arriving at the stops. Considering the differential comfort level experienced by sitting and standing passengers, Hamdouch and Lawphongpanich [13] and Hamdouch et al. [14] proposed equilibrium conditions as variational inequalities that involve a vector-valued function of the expected strategy cost. The method of successive averages (MSA) was applied to solve the model. In addition to the capacity limitation, the supply is not stable. Hamdouch et al. [15] considered uncertainties in supply and proposed an analytical model to record the stochastic nature of transit schedules. The uncertainties are considered as the covariances of travel time between the links in a space-time graph. An MSA-based algorithm was used to solve the aforementioned problem. However, demand and supply uncertainties significantly affected the passenger behavior. Zhang et al. [16] modeled this phenomenon using the invehicle congestion parameter. A heuristic MSA-based algorithm was applied, and the results showed that the risktaking attitude significantly impacts the travel mode and departure-time selection of passengers.

The schedule-based assignment could explain the passenger behavior in detail, but it is relatively complex and time consuming owing to the involvement of time-space path search and dynamic simulation. However, in metropolitans such as Beijing and Tokyo, when passengers planning their trip, they do not have to target to the specific train service because the train frequency is relatively high. Especially in the peak hour, the minimal headway is reduced to $90 \mathrm{~s}$. Meanwhile, there is only one kind of service on the transit line, which means all trains on the same line stop at the same stations. In this situation, passengers do not have to consider timetables or train departure times before trying to catch the trains. Moreover, the train arrival times are announced beforehand for passengers to plan their travel. Passengers can switch their previous paths at some stations to travel more quickly or comfortably. This kind of behavior correlates with the frequency-based assignment assumption.

The frequency-based assignment is commonly used for transit and urban network planning, where the frequency is fixed and high [17]. It is assumed that the passengers may board the first attractive train when they arrived at the stop, which is named the optimal strategy [18] or shortest hyperpath [19]. However, owing to busy transit networks, systems get overloaded and highly congested. As a result, passengers who have missed the first train may have to wait for the second or third attractive train. To demonstrate this phenomenon, De Cea and Fernández [20] proposed the effective frequency, which is linearly related to equivalent average waiting time index, to correctly model the impact of congestion. Since then, several researchers have tried their best to focus on the frequency assignment with capacity constraints [21]. Cepeda et al. [22] considered that travel time is related to the passenger flow. They formulated an equivalent optimization problem with a new characterization of equilibria, which vanished in the computable gap function. Schmöcker et al. [23] modeled the capacity constraint with the "fail-to-board" probability and searched the hyperpath. The Markov network loading process was proposed for passengers who failed to board the first train and reconsidered their selection of routes. Disabled people and pregnant women cared more about the travel experience. Schmöcker et al. [24] introduced a "fail-to-sit" probability, according to which passengers would follow the priority rule that standing on-board passengers would occupy any available seats of alighting passengers before newly boarding passengers do. Passenger behavior is sensitive to perturbations when it comes to running times or service frequencies [25], and some systems provide online information on predicted arrival times. The frequency-based transit assignment model that considers online information can significantly reduce the overall travel time [26]. They tried to proposed capacity limitation with the mathematical formula and transform the model into a new optimization model.

Compared with the schedule-based assignment, the frequency-based assignment could solve the problem more quickly and also demonstrate passenger behavior. It would be interesting if we can combine the merits of the realism of schedule-based methods and the simplicity of frequencybased methods in a single framework to find a balance between accuracy and computational efficiency [27, 28]. At the same time, it is necessary to consider the capacity limitation during the model and make the model into a more practical way.

Therefore, this study considered capacity constraints for the classic optimal strategy model. Moreover, a CVX [29] (for Disciplined Convex Programming) simulation algorithm, which simulates the passenger behavior based on the timetable, has been proposed to solve the problem. The paper is arranged as follows: Section 2 describes the parameters and basic terminologies that will be used in the study. Moreover, a service network has been proposed as the base network for this study. Section 3 proposes the optimal strategy model with the capacity constraints, and the effective frequency has been used to enable the model. Section 4 proposes the CVX simulation algorithm to solve the model proposed in Section 3. Section 5 describes the application of the optimal strategy model and the CVX algorithm to the Beijing Subway Network to verify the feasibility of the model. Section 6 concludes the paper and discusses future related studies.

\section{Notation and Service Network}

2.1. Notation. We first list all the involved notations and decision variables (also mentioned in the aforementioned discussion) in Table 1.

2.2. Service Network. The transit network is the base of the transit assignment process. In the frequency-based assignment model, the network is based on physics lines, which means the physics track. Todiscribe passenger behavior in detail, we demonstrate a service network that comprises passenger behavior and train service links.

To simplify the network illustration, we consider a single direction. As shown in Figure 1(a), there are two physics lines: Line 1 and Line 2. Line 1 serves stations A, B, and E. Line 2 serves stations B, C, and D. Station B is a transfer 
TABLE 1: Notations used in this study.

\begin{tabular}{|c|c|}
\hline Notation & Explanation \\
\hline $\begin{array}{l}G=(E, I, T) \\
K\end{array}$ & $\begin{array}{c}\text { Network } G \text { with nodes } I \text {, link } E \text {, and time slice set } T \\
\text { Passenger set }\end{array}$ \\
\hline$E_{a, t}^{+}$ & Links from node $a$ at time $t$ \\
\hline $\bar{E}_{t}^{*}$ & Optimal strategy at time $t$ \\
\hline$v_{a}^{t}$ & Passenger volume at node $a$ and time $t$ \\
\hline$v_{a}^{t}$ & The total incoming passengers at node $a$ \\
\hline $\begin{array}{l}\operatorname{In}_{a} \\
\alpha\end{array}$ & $\begin{array}{l}\text { The entry passenger at node } a \text {, which generated at node } a \\
\text { Calibration parameters, which depends on the distribution assumed for buses headways and passenger arrival times. }\end{array}$ \\
\hline$c_{(a, b)}^{t}$ & Link $(a, b)$ travel time at time $t$ \\
\hline$f_{(a, b)}^{t}$ & Service frequency for link $(a, b)$ at time $t$ \\
\hline$v_{(a, b)}^{t}$ & Passenger volume for link $(a, b)$ at time slice $t$ \\
\hline $\bar{f}_{(a, b)}^{t}$ & Train valid frequency \\
\hline $\operatorname{Cap}_{(a, b)}^{t}$ & Capacity at link $(a, b)$ at time slice $t$ \\
\hline $\begin{array}{l}\eta \\
c_{e}^{t}\end{array}$ & $\begin{array}{c}\text { Maximal loading factor, which is usually set as } 120 \% \text { or } 130 \% \\
\text { Best value for link } e \text { at time } t\end{array}$ \\
\hline$u_{k}$ & Total travel time for passenger $k$ from automated fare collection (AFC) data \\
\hline$c_{e 0}^{t}$ & Minimal travel time for link $e$ at time $t$ \\
\hline$b^{k, t}$ & Total waiting time calculated from the AFC data with a given path for passenger $k$ at time $t$ \\
\hline $\begin{array}{l}x_{(k, b)}^{t} \\
\delta_{e}^{k, t} \\
\gamma_{e}^{k, t}\end{array}$ & $\begin{array}{c}\text { Binary variable, whether link }(a, b) \text { is recorded in the optimal strategy or not } \\
\text { Binary variable, whether link } e \text { is selected by passenger } k \text { at time } t \\
\text { Binary variable, whether link } e \text { is the none-waiting link or not }\end{array}$ \\
\hline
\end{tabular}

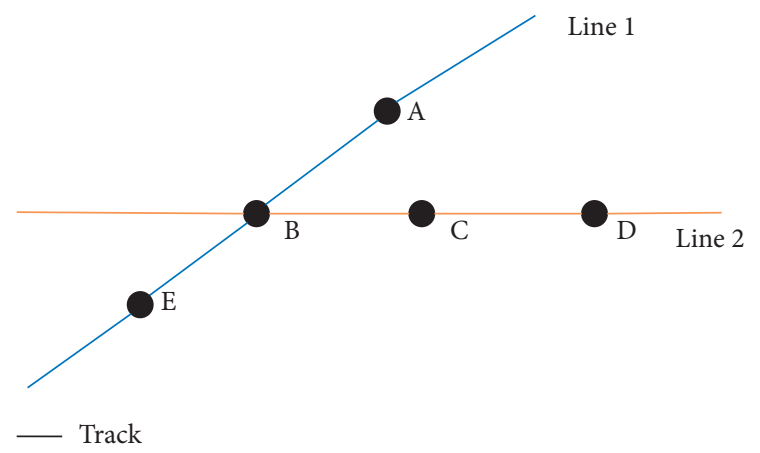

(a)

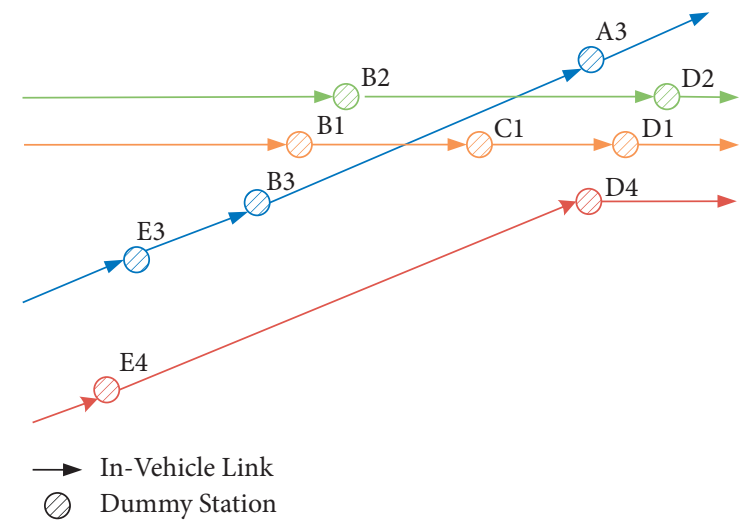

$\oslash$ Dummy Station

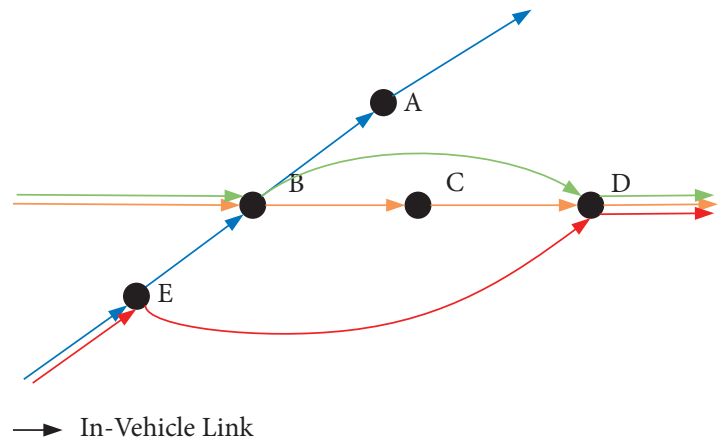

(b)

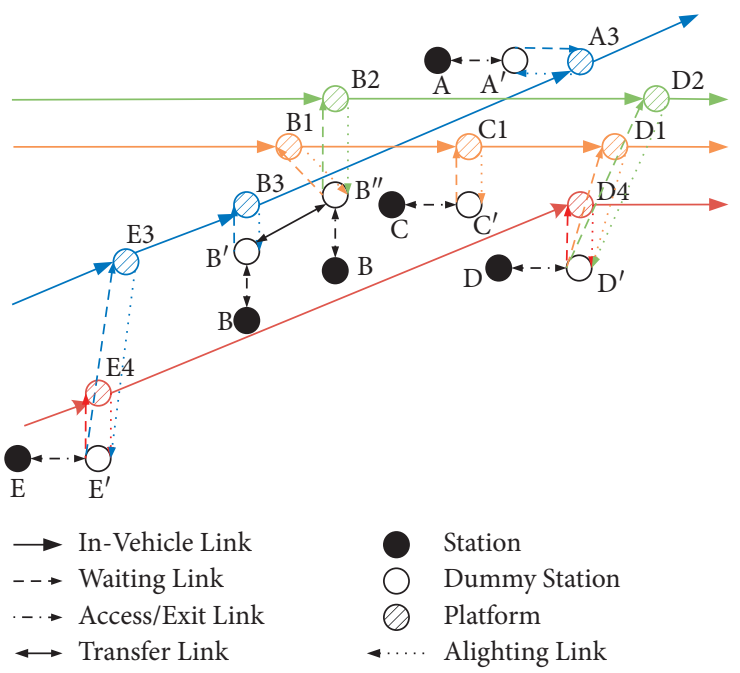

(d)

FIGURE 1: Transit service topology network: (a) physical network, (b) operation service, (c) frequency-based network, and (d) service network. 
station. There are four kinds of transit services in the demo network, which are as follows (Figure 1(b)): stop-stop services denoted by blue and orange colors, skip-stop service denoted by green, and cross-line service denoted by red. In the frequency-based network, the stations are duplicated according to the transit services shown in Figure 1(c). Considering passenger behaviors such as waiting, boarding, and alighting, more links are added to the frequency-based network, which is defined as the service network here. There are five kinds of links: entry-egress links, waiting links, boarding-alighting links, in-vehicle links, and transfer links (Figure 1(d)).

The service network can detail the behavior of passengers from entrance to exit. We take the movement of a passenger from Station $\mathrm{C}$ to Station $\mathrm{D}$ as an example. The passenger reaches node $C$ and walks to the dummy node $C^{\prime}$, and he waits for trains at the waiting link $C^{\prime}-C 1$. When the train arrives, he can take the in-vehicle link C1-D1. He/she gets off when the train arrives at D1, following the alighting link D1- $\mathrm{D}^{\prime}$, and walks out of the station following $\mathrm{D}^{\prime}-\mathrm{D}$.

2.3. Link Cost and Frequency. Each link has two parameters: link frequency and link cost. Link frequency represents the service frequency in which a passenger has to wait for the link. The link cost is the travel time or walking time spent on this link. The service topology network can be classified into two categories: the fixed link frequency and the flexible link frequency. The access and exit, boarding-alighting, in-vehicle, and transfer links belong to the fixed link frequency, where passengers can be served immediately when they reach to the start point of these link. The frequency was set to be infinite. The cost of transfer links, access, and exit links are based on the link walking time, which could be calibrated by field research. The cost of in-vehicle links comes from the timetable, which is the running time between the successive stations. We consider the boarding and alighting can be finished in a quick time and these link costs are set to be 0 . For waiting links, the waiting time depends on the arrival distribution of trains and passengers. When the frequency of passengers follows a uniform distribution and the trains arrive evenlyand without capacity limitation, the waiting link frequency is half of the train frequency. The waiting link cost is 0 .

\section{Optimal Strategy with Capacity Constraints}

In the normal optimal strategy model [2], it is assumed that there is no capacity limitation for the service network. However, in Beijing and Tokyo, the metro systems serve over 10 million passengers every day. In peak hours, the train capacity limitation is a huge obstacle for the passenger boarding process. Based on this, we have generated a timebased transit network $G=(E, I, T)$, where $T$ denotes the set for time slice $t$. We have introduced the capacity limitation constraint, shown in the last equations in F1, which is to minimize the total travel time of all passengers for all links. The train capacity changes or gets updated based on time and stations. The updated optimal strategy model (F1) with capacity constraint variables is shown below:

$$
\begin{aligned}
& \text { (F1) Min } \sum_{(a, b) \in E_{t}} c_{(a, b)}^{t} v_{(a, b)}^{t}+\sum_{a \in I} \alpha \frac{v_{a}^{t}}{\sum_{(a, b) \in E_{a, t}^{+}} f_{(a, b)}^{t} x_{(a, b)}^{t}}, \\
& \text { s.t. }\left\{\begin{array}{l}
v_{(a, b)}^{t}=\frac{f_{(a, b)}^{t} x_{(a, b)}^{t}}{\sum_{(a, b)^{\prime} \in E_{a, t}^{+}} f_{(a, b)}^{t} x_{(a, b)^{\prime}}^{t}} v_{a}^{t},(a, b) \in E_{a, t}^{+}, \quad a \in I, \\
v_{a}^{t}=\sum_{(a, b) \in E_{a, t}^{-}} v_{(a, b)}^{t}+\operatorname{In}_{a}, \quad a \in I, \\
x_{(a, b)}^{t}=\left\{\begin{array}{l}
0, \quad \text { if }(a, b) \notin \bar{E}_{t}^{*}, \\
1, \quad \text { if }(a, b) \in \bar{E}_{t}^{*}, \\
v_{a}^{t} \geq 0, \quad a \in I, \\
v_{(a, b)}^{t} \leq \eta \operatorname{Cap}_{(a, b)}^{t} .
\end{array}\right.
\end{array}\right.
\end{aligned}
$$

We have applied effective frequency $\bar{f}_{(a, b)}^{t}$, which is related to the train capacity, to simplify the model. For instance, we assume that the passenger follows a uniform distribution, the train arrives evenly, and the service frequency is $3 \mathrm{~min}$. When the train capacity is not limited, the waiting time of the passenger is $1.5 \mathrm{~min}$. However, when there are many passengers on the platform and the train capacity is limited, a passenger may wait for the following trains to board on. In this condition, the waiting time is $4.5 \mathrm{~min}$, and the valid train frequency is $9 \mathrm{~min}$. Considering the effective frequency, the new model (F2) can be rewritten as 


$$
\begin{aligned}
& \text { (F2) Min } \sum_{(a, b) \in E_{t}} c_{(a, b)}^{t} v_{(a, b)}^{t}+\sum_{a \in I} \alpha \frac{v_{a}^{t}}{\sum_{(a, b) \in E_{a, t}^{+}} \bar{f}_{(a, b)}^{t} x_{(a, b)}^{t},} \\
& \operatorname{s.t.}_{(a, b)}^{t}=\frac{\bar{f}_{(a, b)}^{t} x_{(a, b)}^{t}}{\sum_{(a, b)^{\prime} \in E_{a, t}^{+}} \bar{f}_{(a, b)}^{t} x_{(a, b)}^{t}} v_{a}^{t},(a, b) \in E_{a, t}^{+}, \quad a \in I, \\
& v_{a}^{t}=\sum_{(a, b) \in E_{a, t}^{-}} v_{(a, b)}^{t}+\operatorname{In}_{a}, \quad a \in I, \\
& x_{(a, b)}^{t}=\left\{\begin{array}{l}
0, \quad \text { if }(a, b) \notin \bar{E}_{t}^{*}, \\
1, \quad \text { if }(a, b) \in \bar{E}_{t}^{*}, \\
v_{a}^{t} \geq 0, \quad a \in I .
\end{array}\right.
\end{aligned}
$$

The effective frequency has relaxed the transportation capacity constraints. The reformed model shares the same structure as the initial optimal strategy model, which implies that the model can be solved using the searching algorithm proposed by Spiess and Florian [18]. As the effective frequency cannot be directly obtained from the timetable, we have proposed a simulated CVX algorithm to obtain the effective frequency for each waiting link.

\section{Passenger Simulation Process and Valid Train Frequency Updating Model}

4.1. Simulated CVX Algorithm. The algorithm flow chart is shown in Figure 2. We cut the simulation time slice by $15 \mathrm{~min}$. In the simulated CVX algorithm, there are two stop criteria. When the simulation time converges to the actual travel time, it implies that the simulation can represent the real situation. Thus, we can stop the simulation process. When the waiting link cost converges compared with the last iteration, the optimal strategy converges, and the simulation stops. The simulation will stop when it meets any of the aforementioned criteria.

The simulated CVX algorithm contains two important parts. The first one is the multi-agent simulation for the optimal strategy, which simulates the passenger behavior based on the optimal strategy and train capacity constraints. The second is the link-updating process to find the optimal value for the waiting link.

4.2. Simulation Using the Capacity-Limited Optimal Strategy. Agent-based simulation for transit services is not a new method [30-32]. The passenger behavior and timetable are set in the agent. For the previous research, the passenger path is assigned to each passenger agent. The passenger agent will follow the given path to finish its trip in the network. Based on the boarding, alighting, and transfer behaviors, the passenger agents and vechicle agents interact with each other.

Based on the previous research, we have designed the passenger and vehicle agents, denoted by red (left part) and blue (right part) in Figure 3. In this simulation process, passengers at each station first search for the optimal strategy. The passengers will follow the strategy when moving toward the destination. They can board the first train or transfer to the following trains according to the optimal strategy. When transferring to other train services, the train capacity should be considered to determine whether the passengers can board the train.

4.3. Link-Updating Model for CVX. A closed automated fare collection (AFC) system obtained an accurate total travel time for each passenger, which can help approach the realtime effective frequency. The least-squares link-updating model P1 is proposed as follows. The objective of P1 is to minimize the gap between the total calculated travel time and the total actual travel time.

$$
\begin{aligned}
& (P 1) \min \sum_{k=1}^{K}\left(\sum_{e \in p\left(\bar{E}_{i}^{t}\right)} c_{e}^{t}-u_{k}\right)^{2}, \\
& \text { s.t. } \quad c_{e}^{t} \geq c_{e 0}^{t}, e \in p\left(\bar{E}_{i}^{t}\right),
\end{aligned}
$$

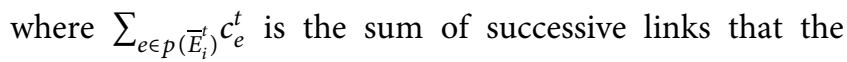
passenger used in the network to finish their trip and $\delta_{e}^{k, t}$ is the selecting index that represents whether the link is selected. P1 can be rewritten as P2, as given below:

$$
\begin{aligned}
& \text { (P2) } \min \sum_{K}\left(\sum_{e \in E} \delta_{e}^{k, t} c_{e}^{t}-u_{k}\right)^{2}, \\
& \text { s.t. }\left\{\begin{array}{l}
c_{e}^{t} \geq c_{e 0}^{t}, \quad e \in E, \\
\delta_{e}^{k, t}= \begin{cases}1, & e \in p\left(\bar{E}_{i}^{t}\right), \\
0, & \text { Otherwise, }\end{cases}
\end{array}\right.
\end{aligned}
$$

where $\sum_{e \in E} \delta_{e}^{k, t} c_{e}^{t}$ defines the total travel time simulated for passenger $k$. As discussed before, all the link costs, except for waiting links, are constant. The total travel time gap can be set as the time gap between the simulation waiting time and real waiting time. Thus, the model can be rewritten as P3.

$$
\begin{aligned}
& \text { (P3) } \min \sum_{K}\left(\sum_{a \in E^{\text {Barding }}} \delta_{e}^{k, t} c_{e}^{t}-\left(u^{k, t}-\sum_{e^{\prime} \in\left\{p\left(\bar{E}_{i}^{t}\right)-E^{\text {Baarding }}\right\}} \gamma_{e^{\prime}}^{k, t} c_{e^{\prime}}^{t}\right)\right)^{2} \text {, } \\
& \text { s.t. }\left\{\begin{array}{l}
\delta_{e}^{\delta_{e}^{t} \geq 0,}= \begin{cases}1, & e \in\left\{p\left(\bar{E}_{i}^{t}\right) \cap E^{\text {Boarding }}\right\}, \\
0, & \text { Otherwise, }\end{cases} \\
\gamma_{e}^{k, t}= \begin{cases}1, & e \in\left\{p\left(\bar{E}_{i}^{t}\right)-E^{\text {Boarding }}\right\}, \\
0, & \text { Otherwise. }\end{cases}
\end{array}\right.
\end{aligned}
$$




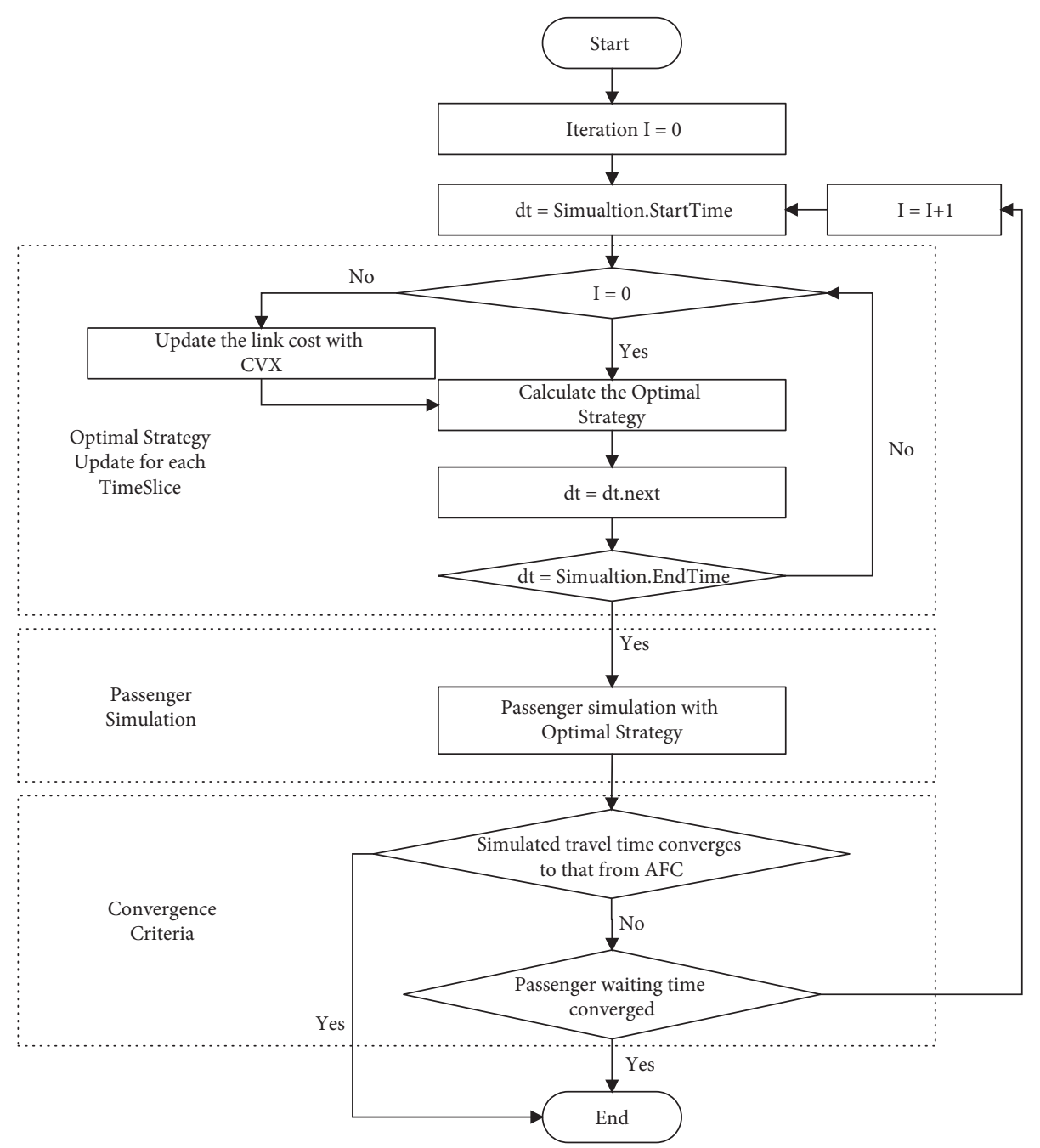

FIgURE 2: Dynamic simulation process for Beijing subway based on the optimal strategy.

The model complexity decreases from all the links to waiting links, and only the non-negative constraint remains. To simplify the model, $b^{k, t}$ is introduced, which is the total waiting time calculated based on the AFC data with a given path.

$$
b^{k, t}=u^{k, t}-\sum_{e^{\prime} \in\left\{p\left(\bar{E}_{i}^{t}\right)-E^{\text {Bording }}\right\}} \gamma_{e^{\prime}}^{k, t} c_{e^{\prime}}^{t}
$$

The matrix formulation of $\mathrm{P} 3$ is shown below:

$$
\begin{aligned}
& (P 4) \min \left(\|A C-B\|_{2}\right)^{1 / 2}, \\
& \text { s.t. } C \geq \overrightarrow{0} .
\end{aligned}
$$

where $C$ is the column for all waiting links in the network and $A$ is the $k \times n$ matrix. For each element in $A, a_{i, j}$ is a dual variable, 1 denotes passenger $i$, and waiting link $j$ is used in this simulation. $B$ is the travel time gap for $k$ passengers. Considering the monotonicity of 2-norm, $\min (\| A C-$ $\left.B \|_{2}\right)^{1 / 2}$ equals $\min \|A C-B\|_{2}$. The current waiting link update model is shown as P5.

$$
\begin{aligned}
& (P 5) \min \|A C-B\|_{2}, \\
& \text { s.t. } C \geq \overrightarrow{0} .
\end{aligned}
$$

The new model has a convex optimization problem and can be solved using CVX, which was proposed by Prof. Stephen Boyd and Dr. Michael Grant from Stanford University [22]. The link-updating process is shown in Figure 4.

\section{Application for Beijing Subway}

5.1. Data in Beijing Subway. Data used in the empirical study are listed in Table 2. We have used one-week AFC data (December 2016) of the Beijing Subway. During that time, there were 17 lines serving more than 10 million passengers every day with more than 8000 train services. The majority of line headways ranged from 2 to $5 \mathrm{~min}$. In the peak hour, the headway could reach $90 \mathrm{~s}$. After applying the service topology construction method, we gathered 96974 links, and every link is labeled with cost and frequency. Based on the annual report of the Beijing Subway, the on-time rate is $>99.9 \%$, and we use the timetable as the base document to initialize the train frequency. 

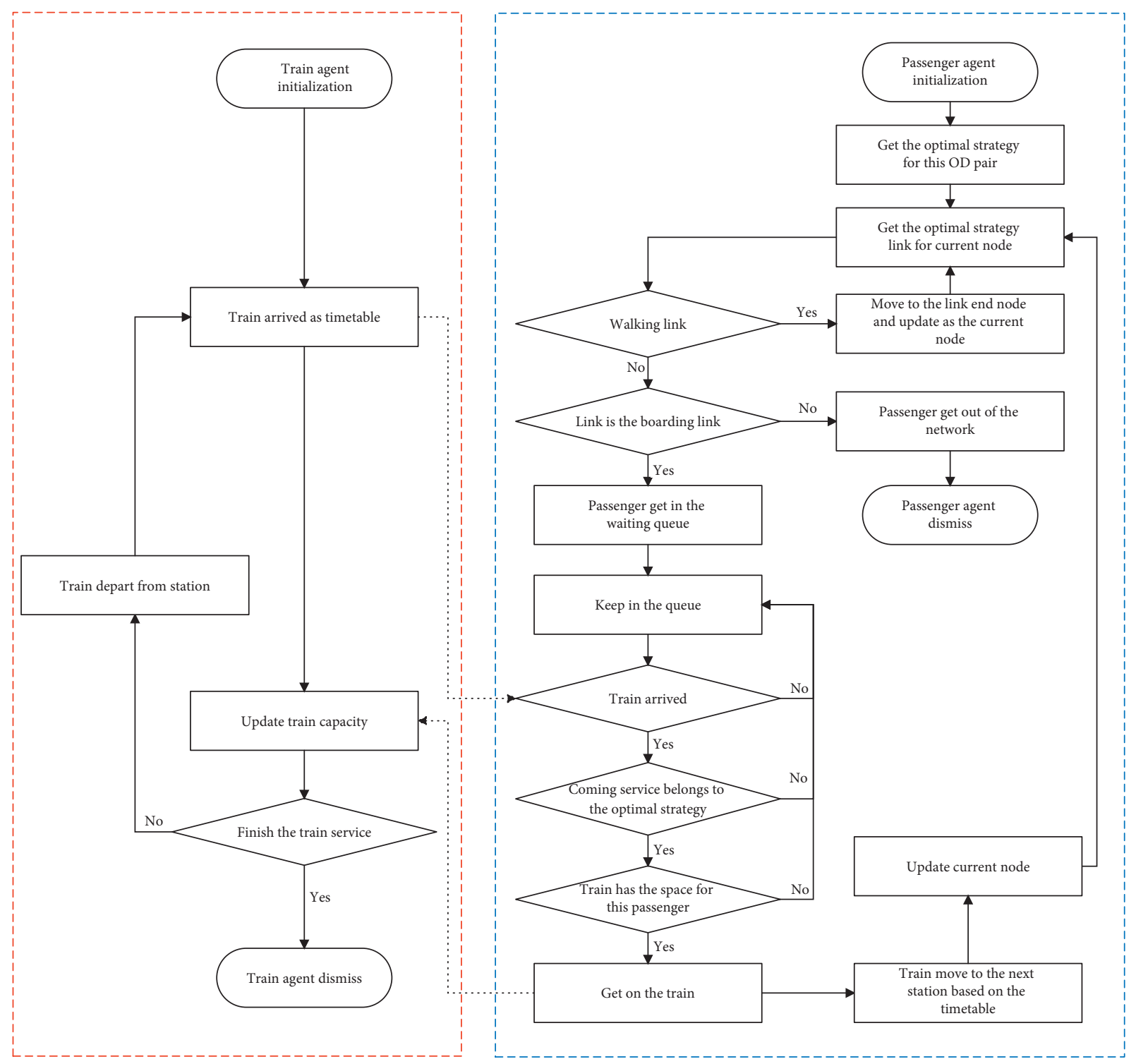

FIGURE 3: Interaction between passenger and vehicle agent.

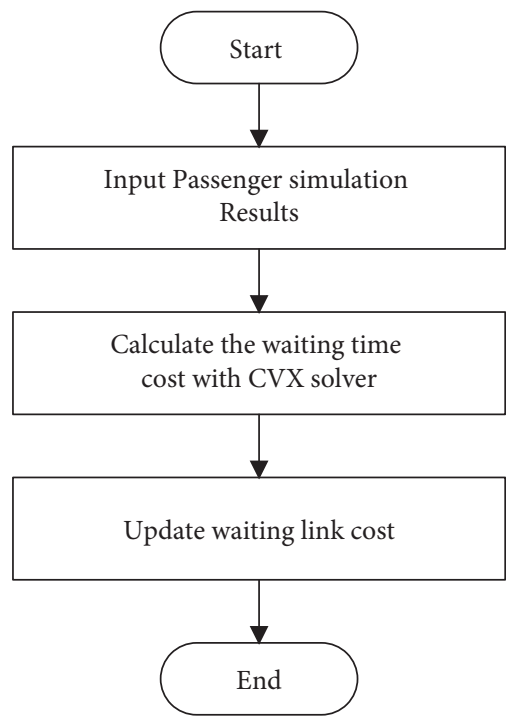

FIGURE 4: Updating process for waiting link. 
TABLE 2: Dataset in the empirical study.

\begin{tabular}{|c|c|c|}
\hline Dataset & Fields & Explanation \\
\hline AFC & $\begin{array}{l}\text { Card ID } \\
\text { O station } \\
\text { Entry time } \\
\text { D station } \\
\text { Exit time }\end{array}$ & $\begin{array}{c}\text { Unique number that could be taken as the passenger ID } \\
\text { Boarding station ID } \\
\text { Access time to the boarding station } \\
\text { Alighting station ID } \\
\text { Exit time from the alighting station }\end{array}$ \\
\hline Link & $\begin{array}{l}\text { Link ID } \\
\text { LinkType } \\
\text { Link cost } \\
\text { Link frequency } \\
\text { Day of week } \\
\text { Time of day }\end{array}$ & $\begin{array}{c}\text { Unique number that could be taken as the link ID } \\
\text { Represent the link belongs to the walking/transfer/boarding or alighting link } \\
\text { Link travel time } \\
\text { Service frequency for the link } \\
\text { Workday or weekend } \\
\text { Peak hour and off-peak hour }\end{array}$ \\
\hline Timetable & $\begin{array}{l}\text { Train no. } \\
\text { StationID } \\
\text { Train loop } \\
\text { Arriving time } \\
\text { Departure time }\end{array}$ & $\begin{array}{c}\text { Train number } \\
\text { Served station for a specific train, ordered by service sequence } \\
\text { Two terminal stations of the loop } \\
\text { Arriving time at each station } \\
\text { Departure time at each station }\end{array}$ \\
\hline
\end{tabular}

5.2. Results Validation. We focus on passenger behavior during the peak hours, 7:00-9:00 AM. The simulation starts at 6:00 AM to warm up the system and ends at 10:00 AM. The simulation process converges to the waiting time criterion after 23 iterations. The validation results are analyzed as follows:

5.2.1. Verification of Total Travel Time. Test samples are simulated results of 7:30 to 8:30 AM. For every $15 \mathrm{~min}$, we have compared the real travel time with the simulated travel time using mean relative error (MRE) and mean absolute relative error (MARE).

$$
\begin{aligned}
\operatorname{MRE} & =\frac{1}{n} \sum_{i=1}^{n} \frac{\left(T_{i}^{s-\text { Exit }}-T_{i}^{a-\text { Entry }}\right)-\left(T_{i}^{a-\text { Exit }}-T_{i}^{a-\text { Entry }}\right)}{\left(T_{i}^{s-\text { Exit }}-T_{i}^{a-\text { Entry }}\right)}, \\
\text { MARE } & =\frac{1}{n} \sum_{i=1}^{n} \frac{\left(T_{i}^{s-\text { Exit }}-T_{i}^{a-\text { Entry }}\right)-\left(T_{i}^{a-\text { Exit }}-T_{i}^{a-\text { Entry }}\right) \mid}{\left(T_{i}^{s-\text { Exit }}-T_{i}^{a-\text { Entry }}\right)} .
\end{aligned}
$$

where $T_{i}^{s-\text { Exit }}$ is the simulated exit time for passenger $i$. $T_{i}^{a-E x i t}$ and $T_{i}^{a-\text { Entry }}$ are the exit and entry times recorded in the AFC system, respectively. The MRE and MARE results are listed in Table 3.

Based on the test period, MRE is $\sim 7 \%$, and MARE is $\sim 10 \%$. Considering the fluctuation in walking time of different passengers, we accept the simulation results.

\subsubsection{Verification of Typical Origin-Destination (OD) Pair.} Considering the diverse travel times among different OD pairs, we have selected typical OD pairs, which have a high passenger volume of $250-400$ in $15 \mathrm{~min}$. These OD pairs comprise large residential areas, e.g., Huilongguan and Huoying, and commercial areas, e.g., Xierqi and Fengtai Science Park. To balance the sample OD spatial distribution, we selected some other OD pairs located in the southeast part of the network.
We applied the $F$-test and $T$-test for the total travel time obtained from these OD pairs, as listed in Table 4 . The result indicates that more than $99 \%$ of the OD pairs passed the test. Less than $1 \%$ of the OD pairs failed the test because the OD volume is somewhat minimal, which means that the less travel time sample is not very stable. Thus, the proposed model and algorithm can represent passenger behavior in the Beijing Subway network. Furthermore, we can analyze the passenger behavior using the optimal strategy.

\subsection{Passenger Behavior Analysis Using the Optimal Strategy}

5.3.1. Waiting Time during the Peak Hours in the Morning. Owing to the station entrance limitation, operation plan, and total travel demand, passengers get delayed while reaching the platform and need to wait for trains during the peak hours in the morning. To analyze the waiting time at the platform, we select the stations that has a top entry-in or transfer passenger volume, including the direction. The selected stations and their waiting times are shown in Figure 5.

For the first $15 \mathrm{~min}$, from 7:30 to 7:45 AM, the average waiting time (AWT) is $157 \mathrm{~s}$. The AWT decreases to $123 \mathrm{~s}$ in the next $15 \mathrm{~min}$. This represents the crowd dispersed over time. Considering the Xingong station on Line 4 as an example, the train headway is $120 \mathrm{~s}$. From 7:30 to 7:45 AM, the AWT increases to $297 \mathrm{~s}$, which implies that passengers need to wait for three headways until they board the arriving train. After $30 \mathrm{~min}$, the AWT deceases to $74.3 \mathrm{~s}$, approximately half of the headway, which implies that the passenger can board the next train when it arrives. The condition of the Shuangjing station denoted by Line 10 is the opposite. The headway is $120 \mathrm{~s}$. In the first $15 \mathrm{~min}$, the AWT is $84 \mathrm{~s}$, and it increases to $158.7 \mathrm{~s}$ after $30 \mathrm{~min}$, which means that passengers have to wait for another train. This could be summarized as the rule that most of the commuters live in a rural area and work downtown. During the peak hours in the morning, the main stations, e.g., Tiangngyuan, Xingong, and Shahe, pertaining to waiting 
TABLE 3: MRE and MARE results for every $15 \mathrm{~min}$.

\begin{tabular}{llc}
\hline Period (AM) & MRE\% & MARE\% \\
\hline $7: 30-7: 45$ & -6.094 & 9.5164 \\
$7: 45-8: 00$ & -7.148 & 10.2293 \\
$8: 00-8: 15$ & -7.026 & 10.4602 \\
$8: 15-8: 30$ & -6.133 & 10.0834 \\
\hline
\end{tabular}

Table 4: $T$-test and $F$-test for OD travel time.

\begin{tabular}{|c|c|c|c|c|c|c|c|c|c|}
\hline \multirow[t]{2}{*}{ Origin } & \multirow[t]{2}{*}{ OD pairs destination } & \multicolumn{2}{|c|}{$\begin{array}{c}7: 30-7: 45 \\
\text { AM }\end{array}$} & \multicolumn{2}{|c|}{$\begin{array}{c}7: 45-8: 00 \\
\text { AM }\end{array}$} & \multicolumn{2}{|c|}{$\begin{array}{c}8: 00-8: 15 \\
\text { AM }\end{array}$} & \multicolumn{2}{|c|}{$\begin{array}{c}8: 15-8: 30 \\
\text { AM }\end{array}$} \\
\hline & & $V$ & $P$ & $V$ & $P$ & $V$ & $P$ & $V$ & $P$ \\
\hline TiantongyuanNorth & Huixinxijie Beikou & 88 & $\mathrm{Y}$ & 117 & $\mathrm{Y}$ & 187 & $\mathrm{Y}$ & 122 & $\mathrm{Y}$ \\
\hline Xingong & Xuanwumen & 54 & Y & 77 & $\mathrm{Y}$ & 52 & $\mathrm{~N}$ & 61 & $\mathrm{Y}$ \\
\hline Tuqiao & Sihui & 223 & $\mathrm{Y}$ & 227 & $\mathrm{Y}$ & 184 & $\mathrm{Y}$ & 145 & $\mathrm{Y}$ \\
\hline Shahe University Park & Xierqi & 412 & $\mathrm{Y}$ & 428 & $\mathrm{Y}$ & 437 & $\mathrm{Y}$ & 352 & $\mathrm{Y}$ \\
\hline Huoying & Wudaokou & 162 & $\mathrm{Y}$ & 215 & $\mathrm{Y}$ & 200 & $\mathrm{Y}$ & 160 & $\mathrm{Y}$ \\
\hline Shilihe & Jintaixizhao & 99 & $\mathrm{Y}$ & 196 & $\mathrm{Y}$ & 269 & $\mathrm{Y}$ & 271 & $\mathrm{Y}$ \\
\hline Changyang & Fengtai Science Park & 140 & $\mathrm{Y}$ & 204 & $\mathrm{Y}$ & 354 & $\mathrm{Y}$ & 281 & $\mathrm{Y}$ \\
\hline Caofang & Chaoyangmen & 145 & $\mathrm{Y}$ & 169 & $\mathrm{Y}$ & 141 & $\mathrm{Y}$ & 82 & $\mathrm{Y}$ \\
\hline Libafang & Fengtai Science Park & 89 & $\mathrm{Y}$ & 73 & $\mathrm{Y}$ & 150 & $\mathrm{Y}$ & 92 & $\mathrm{Y}$ \\
\hline Songjiazhuang & Chaoyangmen & 105 & $\mathrm{Y}$ & 95 & $\mathrm{Y}$ & 93 & $\mathrm{Y}$ & 77 & $\mathrm{Y}$ \\
\hline
\end{tabular}

$V$ : OD volume; $P$ : pass the test; Y: yes; N: no.

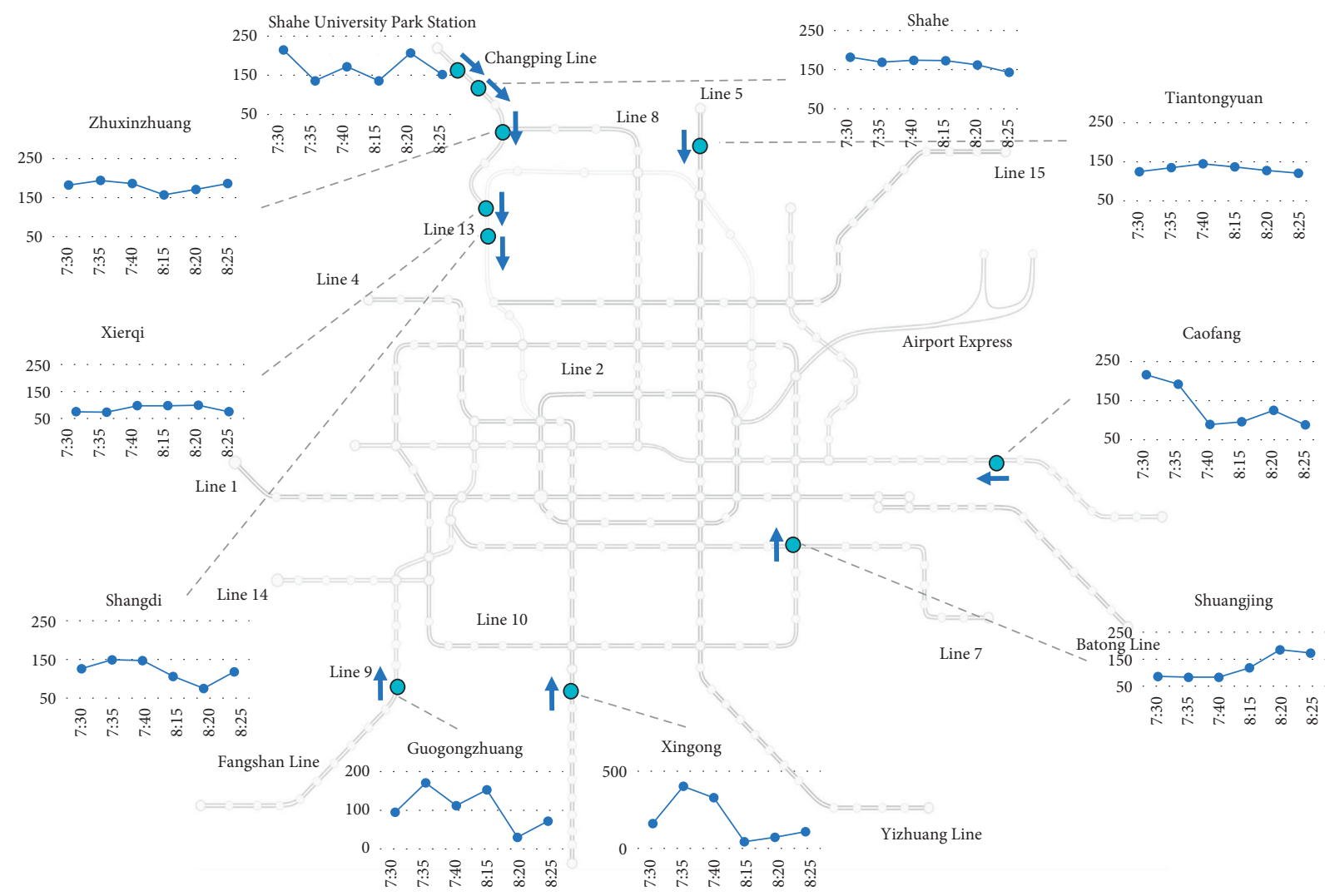

FIgURE 5: Waiting time analysis for stations.

time are distributed in rural lines. Over time, the crowd moves to the central part of the network and downtown stations.
5.3.2. Loading Factor for Different Loops on the Same Line. Line 4-daxing has two loops (Figure 6). The long loop is from Tiangongyuan to Anheqiaobei; both are terminal stations. 


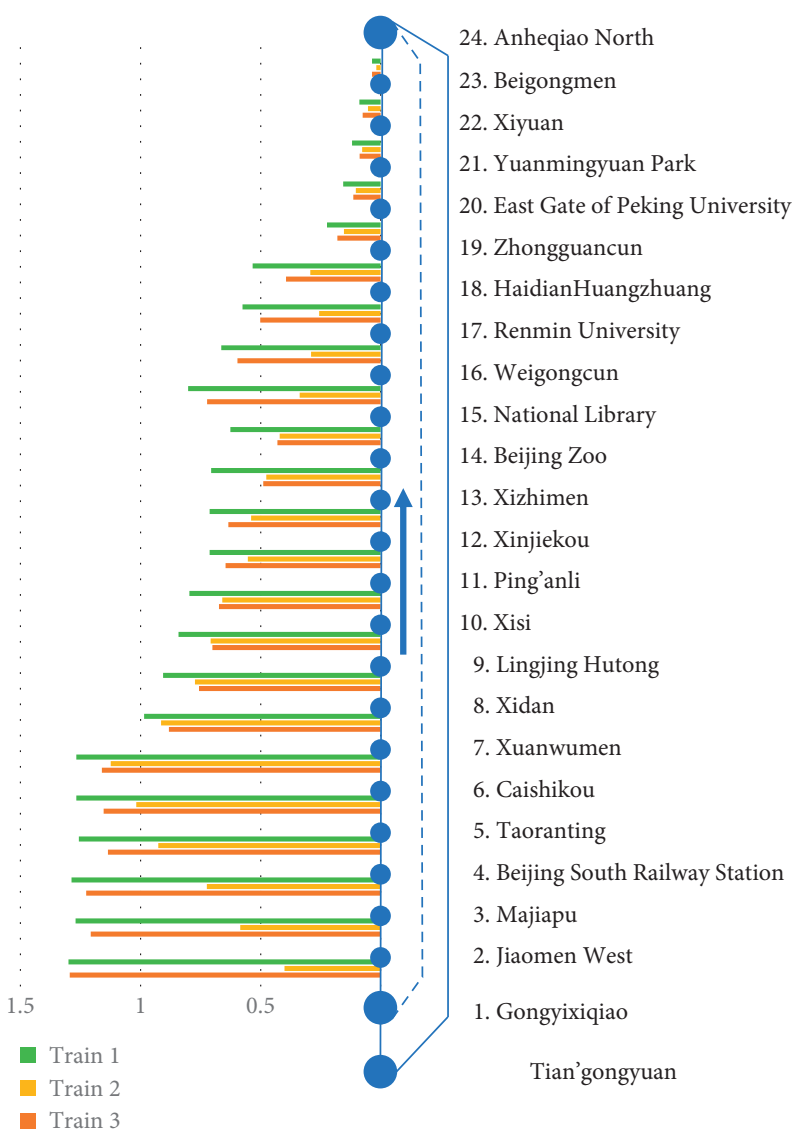

Figure 6: Operation loop of line 4-daxing in Beijing.

The shorter loop (dashed line) is from Gongyixiqiao to Anheqiaobei (Figure 6). To simplify the presentation, stations from Tiangongyuan to Gongyixiqiao have been excluded.

In the morning, many passengers travel from south to north. According to the optimal strategy, the service links from long and short loops are recorded, and passengers can choose a long loop or a short loop. We have selected three successive long-short loop train services. Train 1 and Train 3 , i.e., long-loop services, arrive at Gongyixiqiao at 7:29:06 and 7:32:06 AM, respectively (Figure 6). Train 2, i.e., the shortloop service, arrives at Gongyixiqiao at 7:30:51 AM (Figure 6). The loading factors of the trains at each section from Gongyixiqiao to the terminal station are denoted by bar charts in Figure 6.

The loading factor in the long loop is greater than that in the short loop. A huge difference is observed between Gongyixiqiao and Taoranting (Section ID 1-5). In the stations in this section, passengers can switch between longloop and short-loop trains. Owing to the low frequency of the short loop, which is $10 \mathrm{~min}$ on an average, passengers who board the long-loop train will not switch to the short loop train, even if the short loop has a lower loading factor. This leads to an imbalance between the long and short loops. For future operations, we should apply skip-stop plans with flexible loop operations to balance the passenger flow and reduce their total travel time.
5.3.3. Transfer Volume in the Network. The average transfer index in Beijing is 1.8, which means every passenger has to be transferred 1.8 times before arriving at the destination. Figure 7 represents the top eight transfer stations ordered by the transfer volume. For every $15 \mathrm{~min}$, the transfer volume was over 4000 in average. Similar to the waiting time distribution, the transfer volume decreases with time in the rural area, while the transfer volume increases in the station that is located in the center of the network. In the future operation design, thorough services for some transit lines should be considered to reduce the transfer and total travel time, with the constraints of turn-back and rolling stocks.

From simulation results, it could be found that, in the morning peak hour, both the long waiting time stations and large transfer volume stations are not in the central of the network, which are specific evidences that most of the commuters are living in the rural area. Second, from the time series of waiting time and transfer volume, the peak of the morning peak hour is very intense. Because of the super high demand and the limited capacity, passengers need to wait for the second even third successive train. To better satisfy the passenger demand, the flexible operation solution such as the long-short loop was provided. Compared with the single operation scheme, the long-short loop relieved some operation pressure, but it could be better if other operation scheme could be applied, such as stop-skip pattern. Actually, the Beijing metro operation company keeps trying to 


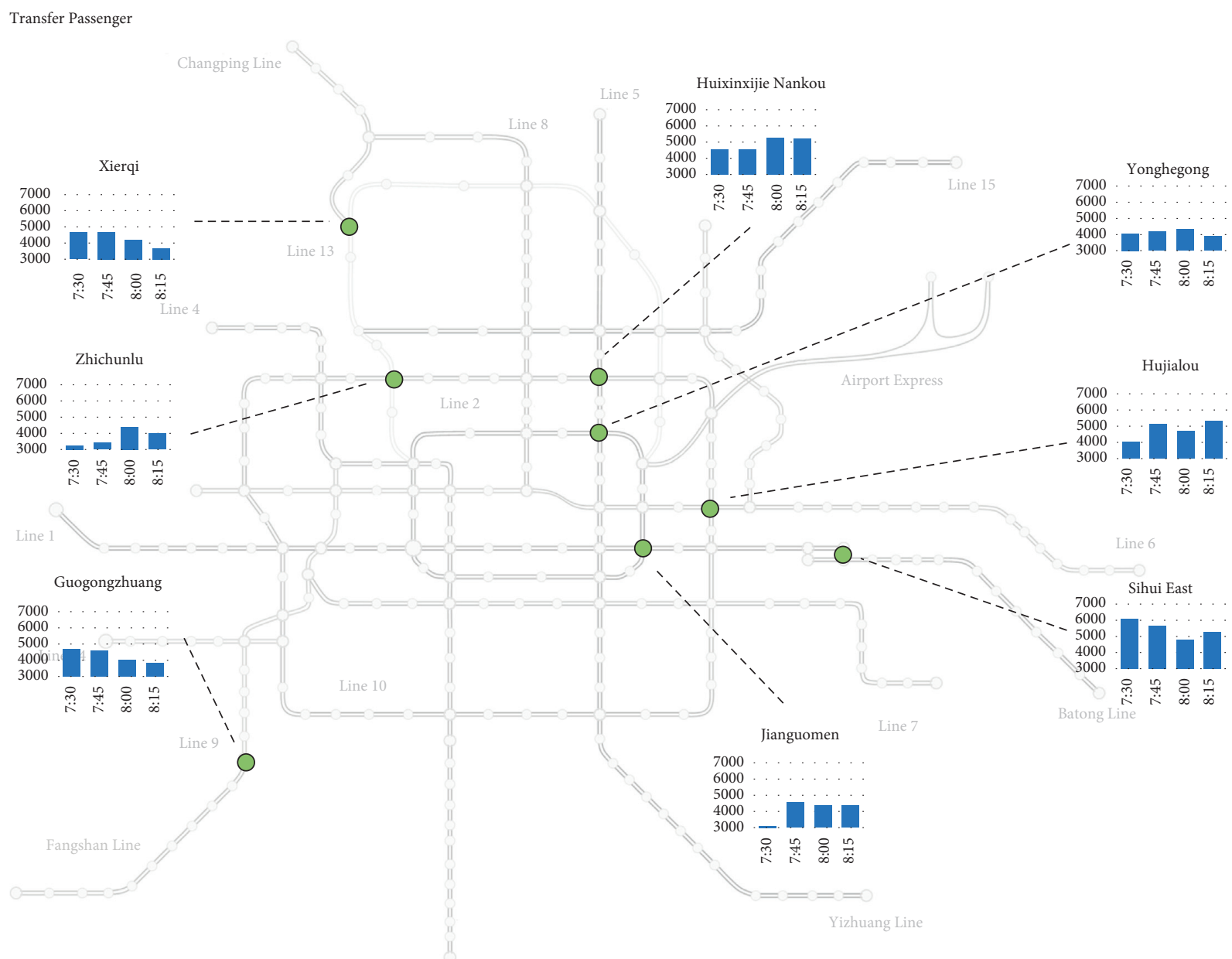

Figure 7: Locations of top eight transfer stations during the peak hours in the morning.

improve the system capacity. During the COVID-19 period in 2020, the more flexible and strong timetables were provided, such as the asymmetry timetable and skip-stop timetable to better serve the tidal passenger flow. At the same time, in order to reduce the passenger transfer time, the trough services between Line 1 and Line Batong are provided. Because of the lack of data, the performance of the new timetables was not analyzed in this research.

\section{Conclusion}

The Beijing Subway has a high and fixed transit service frequency based on the assumption analysis of the frequency-based transit assignment method and optimal strategy. Considering the train capacity limitation in the metro system, this study introduces the capacity constraint for the optimal strategy. To overcome the capacity limitation, a CVX simulation algorithm has been proposed. The empirical study demonstrates that the updated model and algorithm can solve the transit assignment problem for busy transit systems. The results detail the passenger behavior in the network. It is possible to analyze the passenger behavior under complex conditions to provide better solutions.

The main aim of the optimal strategy is to represent passenger behavior. However, some passengers preferred to determine the path in advance. In that case, they seldom change their path during the trip, and the logit model can demonstrate their behavior better than the optimal strategy. Every model has its own assumptions and characteristics. It is better to analyze passengers based on their perspectives.

Meanwhile, the objective of the optimal strategy model is to minimize the total travel time of passengers. However, the decision-making process considers more than total travel time. Moreover, they would consider discomfort or transfer time. Therefore, the transfer time and transfer penalty should be added to the objective function, thereby making the model closer to reality.

\section{Data Availability}

The AFC and timetable used to support the findings of this study were supplied by the project: the metro network operation supervision and forecasting, provided by Transportation Operations Coordination Center and Beijing Jiaotong University, cannot be made freely available. The service link is from https://map.bjsubway.com, which is open to public.

\section{Conflicts of Interest}

The authors declare no conflicts of interest. 


\section{Authors' Contributions}

Conceptualization, K.L. and N.C.; methodology, K.L. and N.C.; formal analysis, K.L; writing-original draft preparation, K.L.; resources, K.L. and N.C.; supervision, N.C.

\section{Acknowledgments}

The authors would like to thank Dr. Alireza Khani from the University of Minnesota and Professor Zijia Wang from Beijing Jiaotong University for helping us edit and Polish the language of this paper. The authors would like to thank Editage (http://www.editage.cn) for English language editing. This work is supported by National Key R\&D Program of China (ID: 2020YFB1600702), Beijing Postdoctoral Research Foundation (Award Number: ZZ2019-118), and Beijing Science and Technology Project (ID: Z191100002519003).

\section{References}

[1] F. Chen, H. Yang, and Y. Yang, "Mas-based model for evaluating train timetables to minimise the waiting time," KSCE Journal of Civil Engineering, vol. 23, no. 3, pp. 13601368, 2019.

[2] Y. Zhou, Y. Wang, H. Yang, and X. Yan, "Last train scheduling for maximizing passenger destination reachability in urban rail transit networks," Transportation Research Part B: Methodological, vol. 129, pp. 79-95, 2019.

[3] Y. Chen, B. Mao, Y. Bai, T. K. Ho, and Z. Li, "Timetable synchronization of last trains for urban rail networks with maximum accessibility," Transportation Research Part C: Emerging Technologies, vol. 99, pp. 110-129, 2019.

[4] Y. Zhu and R. M. P. Goverde, "Dynamic passenger assignment for major railway disruptions considering information interventions," Networks and Spatial Economics, vol. 19, no. 4, pp. 1249-1279, 2019.

[5] L. L. Cheung, Shalaby, A. S. Amer, and Shalaby, "System optimal re-routing transit assignment heuristic: a theoretical framework and large-scale case study," International Journal of Transportation Science and Technology, vol. 6, no. 4, pp. 287-300, 2017.

[6] K. Gkiotsalitis and O. Cats, "Timetable recovery after disturbances in metro operations: an exact and efficient solution," IEEE Transactions on Intelligent Transportation Systems, pp. 1-11, 2020.

[7] N. Papola, F. Filippi, G. Gentile, and L. Meschini, "Schedulebased transit assignment: new dynamic equilibrium model with vehicle capacity constraints," Schedule-Based Modeling of Transportation Networks, vol. 46, pp. 1-26, 2010.

[8] A. Nuzzolo and U. Crisalli, "The schedule-based approach in dynamic transit modelling: a general overview," ScheduleBased Dynamic Transit Modeling: Theory and Applications, vol. 28, pp. 1-24, 2004.

[9] A. Nuzzolo, F. Russo, and U. Crisalli, Transit Network Modelling. The Schedule-Based Dynamic Approach, Collana Trasporti; Franco Angeli, Milan, Italy, 2003.

[10] A. Nuzzolo, F. Russo, and U. Crisalli, "A doubly dynamic schedule-based assignment model for transit networks," Transportation Science, vol. 35, no. 3, pp. 268-285, 2001.

[11] M. H. Poon, S. C. Wong, and C. O. Tong, "A dynamic schedule-based model for congested transit networks,"
Transportation Research Part B: Methodological, vol. 38, no. 4, pp. 343-368, 2004.

[12] A. Nuzzolo, U. Crisalli, and L. Rosati, "A schedule-based assignment model with explicit capacity constraints for congested transit networks," Transportation Research Part C: Emerging Technologies, vol. 20, no. 1, pp. 16-33, 2012.

[13] Y. Hamdouch and S. Lawphongpanich, "Schedule-based transit assignment model with travel strategies and capacity constraints," Transportation Research Part B: Methodological, vol. 42, no. 7-8, pp. 663-684, 2008.

[14] Y. Hamdouch, H. W. Ho, A. Sumalee, and G. Wang, "Schedule-based transit assignment model with vehicle capacity and seat availability," Transportation Research Part B: Methodological, vol. 45, no. 10, pp. 1805-1830, 2011.

[15] Y. Hamdouch, W. Y. Szeto, and Y. Jiang, "A new schedulebased transit assignment model with travel strategies and supply uncertainties," Transportation Research Part B: Methodological, vol. 67, pp. 35-67, 2014.

[16] Y. Zhang, W. H. K. Lam, A. Sumalee, H. K. Lo, and C. O. Tong, "The multi-class schedule-based transit assignment model under network uncertainties," Public Transport, vol. 2, no. 1-2, pp. 69-86, 2010.

[17] L. Meschini, G. Gentile, and N. Papola, "A frequency based transit model for dynamic traffic assignment to multimodal networks," in Proceedings of the 17th International Symposium on Transportation and Traffic Theory, vol. 2, no. 3, pp. 2-3, London, 2007 July.

[18] H. Spiess and M. Florian, "Optimal strategies: a new assignment model for transit networks," Transportation Research Part B: Methodological, vol. 23, no. 2, pp. 83-102, 1989.

[19] S. Nguyen and S. Pallottino, "Equilibrium traffic assignment for large scale transit networks," European Journal of Operational Research, vol. 37, no. 2, pp. 176-186, 1988.

[20] J. De Cea and E. Fernández, "Transit assignment for congested public transport systems: an equilibrium model," Transportation Science, vol. 27, no. 2, pp. 133-147, 1993.

[21] R. Cominetti and J. Correa, "Common-lines and passenger assignment in congested transit networks," Transportation Science, vol. 35, no. 3, pp. 250-267, 2001.

[22] M. Cepeda, R. Cominetti, and M. Florian, "A frequency-based assignment model for congested transit networks with strict capacity constraints: characterization and computation of equilibria," Transportation Research Part B: Methodological, vol. 40, no. 6, pp. 437-459, 2006.

[23] J.-D. Schmöcker, M. G. H. Bell, and F. Kurauchi, "A quasidynamic capacity constrained frequency-based transit assignment model," Transportation Research Part B: Methodological, vol. 42, no. 10, pp. 925-945, 2008.

[24] J.-D. Schmöcker, A. Fonzone, H. Shimamoto, F. Kurauchi, and M. G. H. Bell, "Frequency-based transit assignment considering seat capacities," Transportation Research Part B: Methodological, vol. 45, no. 2, pp. 392-408, 2011.

[25] K. Nökel and S. Wekeck, "Boarding and alighting in frequency-based transit assignment," Transportation Research Record: Journal of the Transportation Research Board, vol. 2111, no. 1, pp. 60-67, 2009.

[26] N. Oliker and S. Bekhor, "A frequency based transit assignment model that considers online information," Transportation Research Part C: Emerging Technologies, vol. 88, pp. 17-30, 2018.

[27] E. Cascetta and P. Coppola, "Assessment of schedule-based and frequency-based assignment models for strategic and operational planning of high-speed rail services," 
Transportation Research Part A: Policy and Practice, vol. 84, pp. 93-108, 2016.

[28] B. Otto, "Aggregation techniques for frequency assignment in public transportation," Public Transport, vol. 11, no. 1, pp. 51-87, 2018.

[29] http://cvxr.com/cvx/.

[30] E. Adamey, A. Kurt, and U. Ozguner, "Agent-based passenger modeling for intelligent public transportation," in Proceedings of the 16th International IEEE Conference on Intelligent Transportation Systems (ITSC 2013), IEEE, The Hague, Netherlands, Oct 2013.

[31] T. Schelenz, Á. Suescun, L. Wikström, and M. Karlsson, "Application of agent based simulation for evaluating a bus layout design from passengers' perspective," Transportation Research Part C: Emerging Technologies, vol. 43, pp. 222-229, 2014.

[32] H. Yin, B. Han, D. Li, J. Wu, and H. Sun, "Modeling and simulating passenger behavior for a station closure in a rail transit network," PLoS One, vol. 11, no. 12, Article ID e0167126, 2016. 